\title{
Stability of Rectification of Iron Porphyrin Molecular Junctions
}

Guoguang Qian, Qi Zhou, and Kim M. Lewis

Department of Physics, Applied Physics, and Astronomy, Rensselaer Polytechnic Institute, Troy, New York 12180, USA

\begin{abstract}
We report rectification from porphyrin molecules ligated to an iron atom. Current-voltage (IV) curves were measured from the molecules using a conductive atomic force microscope (AFM). Molecules were deposited on a substrate from template-stripped gold from $1 \mu \mathrm{M}$ iron porphyrin solution by either a drop-dry or 60 second deposition method. The measured IV curves from the drop-dry samples were stable. Statistical analysis of the IV curves showed that the distribution of the threshold voltages $(0.543 \mathrm{~V}-0.588 \mathrm{~V})$ and rectification ratios (34 42) from the drop-dry samples are confined to a smaller range than the threshold voltages $(0.2 \mathrm{~V}-0.7 \mathrm{~V})$ and rectification ratios $(2.5$ - 162) from the 60 second samples. The fluctuations in the IV curve from the 60 second deposition samples can be explained by local joule heating. The roughness of the topography was analyzed to understand the difference in IV measurements between the two types of samples. The stability of the rectification from the drop-dry samples is attributed to good thermal contact between the AFM tip and the molecules on the substrate.
\end{abstract}

Keywords: Atomic force microscopy; Electrical properties; Interfaces, Nanostructures.

Tel: $+1(518) 2763252$

Fax: +1(518)2762996

Email: qiang2@rpi.edu 


\section{Introduction}

Since Aviram and Ratner proposed the first single molecular rectifier [1] in 1974, a molecular diode has been realized by using a Langmuir-Blodgett (LB) film [2-3] and a self-assembled monolayer [4]. The rectification of the LB film and the monolayer was characterized by placing the film or the monolayer between two electrodes to measure the current versus voltage (IV).[2-4] Similarly, the characterization can be carried out electrochemically by placing the film or monolayer of molecules on a working electrode, and measuring a voltammogram with an electrochemical potentiostat.[5] In both scenarios, the characterization reflected the property of a large number of molecules due to the macro-size dimensions of the electrodes used for the measurement. Recently, the rectification of molecules has been investigated with scanning tunneling microscopy (STM) by positioning molecules between a substrate and an STM tip. [6-8] Due to its nano-scale size of the tip, the STM measured the rectification from a few molecules to a single molecule with a molecular break junction technique.[6] From these measurements the IV curves showed instability induced by local joule heating and electromigration. Also, the irreproducibility of the measurement was observed because the electrical transport properties of the molecules depended on the contact between the molecule and the electrode and this contact changed with each IV curve measured. In order to realize molecular electronics that use molecules as active components, such as storage elements [9] in a circuit, the instability and irreproducibility observed in IV curves must be addressed.

In this study we have experimentally investigated rectification by conductive atomic force microscopy from porphyrin molecules ligated to an iron atom (FeP). We observed stable rectification from molecules deposited by drying drops of $1 \mu \mathrm{M}$ FeP solution on a substrate prepared from template-stripped gold from silicon. This investigation may provide insight into the role that surface roughness at the molecule-electrode interface contributes to device stability and the design of future molecular electronic devices.

\section{Materials and methods}

The gold substrate was prepared using a template-stripped method [10]. A gold ( $\mathrm{Au}$ ) film of $200 \mathrm{~nm}$ was deposited on a silicon (110) wafer by electron beam evaporation. The $\mathrm{Au}$ (99.999\% purchased from International Advanced Materials) was evaporated at a rate of $\sim 1$ $\mathrm{nm} / \mathrm{s}$. A two component adhesive EPO-TEK 377 (purchased from Epoxy Technology) was applied to the surface of the Au film and a glass slide was attached to the epoxy layer. This sample was cured at $150^{\circ} \mathrm{C}$ in an oven for one hour. After curing, the Au film was peeled from the silicon wafer. Atomic force microscopy was completed to determine the flatness of the template-stripped Au surface. The surface was analyzed using average values of the root mean square (RMS). The average RMS value was determined to be $0.160 \mathrm{~nm} \pm 0.039 \mathrm{~nm}$ from 364 selected areas of $0.25 \mu \mathrm{m} \times 0.25 \mu \mathrm{m}$. The same measurement was also performed for gold on mica and gold evaporated on silicon from 50 selected areas of $0.25 \mu \mathrm{m} \times 0.25 \mu \mathrm{m}$ and the average RMS values were $1.09 \mathrm{~nm} \pm 0.38 \mathrm{~nm}$ and $1.13 \mathrm{~nm} \pm 0.08 \mathrm{~nm}$, respectively. Based on these values the template-stripped gold substrate is much flatter than gold on mica and gold evaporated on silicon without using the template-stripped method. In order to 
obtain a fresh $\mathrm{Au}$ surface, the Au film was peeled from the silicon wafer just before depositing molecules onto the surface.

Iron porphyrin [Fe(III) 5,15-di[4-(s-acetylthio)phenyl]-10,20-diphenyl porphine acetate] (hereafter denoted FeP) was purchased from Frontier Scientific Porphyrin Products Division. The molecular concentration of $1 \mu \mathrm{M}$ FeP in toluene solution was prepared by diluting a $1 \mathrm{mM}$ FeP toluene solution.

Two methods were used to deposit the molecules: a drop-dry method and a 60 second deposition method. For the drop-dry method, three drops of $10 \mu \mathrm{L}$ from a $1 \mu \mathrm{M}$ FeP toluene solution were transferred to the center of a $1 \mathrm{~cm} \times 1 \mathrm{~cm} \mathrm{Au}$ substrate using a $10 \mu \mathrm{L}$ pipette. After several minutes, the toluene evaporated and molecules remained on the substrate. For the 60 second deposition method, the substrate was submerged in a $1 \mu \mathrm{M}$ FeP toluene solution for 60 seconds. After the sample was removed from the solution, the substrate was rinsed with toluene several times before it was blow-dried with nitrogen. The sample as prepared is denoted hereafter as the $60 \mathrm{~s}$ sample. For both methods, the FeP molecules were deposited on template-stripped Au substrate from silicon.

\section{Experimental}

The current-voltage (IV) measurements were performed with a PSIA (Park Systems Corporation) XE-120 Atomic Force Microscope (AFM) in current-AFM mode using an external current preamplifier with variable gain. The topography of the samples was obtained with the AFM in contact mode. A gold coated AFM tip (HQ: NSC18/Cr-Au) purchased from MikroMasch with a force constant of $2.8 \mathrm{nN} / \mathrm{m}$ and an approximate radius of $35 \mathrm{~nm}$ was used for both IV measurements and topography scans. A force of $3 \mathrm{nN}$ was applied to the molecules of FeP. The system was allowed at least 20 minutes to become stable before IV curves were measured. The IV was completed using a bias range from $-1 \mathrm{~V}$ to $1 \mathrm{~V}$. The current measured from the FeP molecules was amplified with a current preamplifier (FEMTO DLPCA-200) purchased from PSIA and measured using data acquisition. Measurements were completed at different locations on the surface of the sample. As a control experiment the IV measurement was also performed on a bare gold substrate not exposed to toluene and a gold substrate after submersion in toluene.

\section{Results and discussions}

For the measurements the samples were biased through the substrate instead of the tip; the tip is virtual ground in this experiment. The rectification behavior was observed in the negative current and voltage direction. When the tip was biased as reported in previous work [12] the rectification was observed in the positive voltage regime. For the purpose of convention the current and voltage values of the original plot was inverted.

The IV curve from the drop-dry sample was observed to be stable, reproducible, and in the current range of micro-ampere. In order to demonstrate the stability and reproducibility of the IV measurement from the drop-dry sample, IV measurements from ten cyclic scans between $1 \mathrm{~V}$ and $1 \mathrm{~V}$ were performed and are shown in the Fig. 1(a). These cyclic IV curves were 
measured with a scan step of $16.65 \mathrm{mV}$, a scan rate of $22.22 \mathrm{mV} / \mathrm{s}$, and the measurement lasted about 30 minutes. IV curves from cyclic voltage scans are shown to be stable and reproducible. With the bias voltage scanned from $-1 \mathrm{~V}$ to $1 \mathrm{~V}$, the current is close to zero until the voltage reaches a threshold voltage. Beyond the threshold voltage the current starts to drastically increase. The IV curves can be evaluated by a rectification ratio (RR) that is defined as the absolute value of the ratio of the current at the maximum bias voltage over the minimum bias voltage.
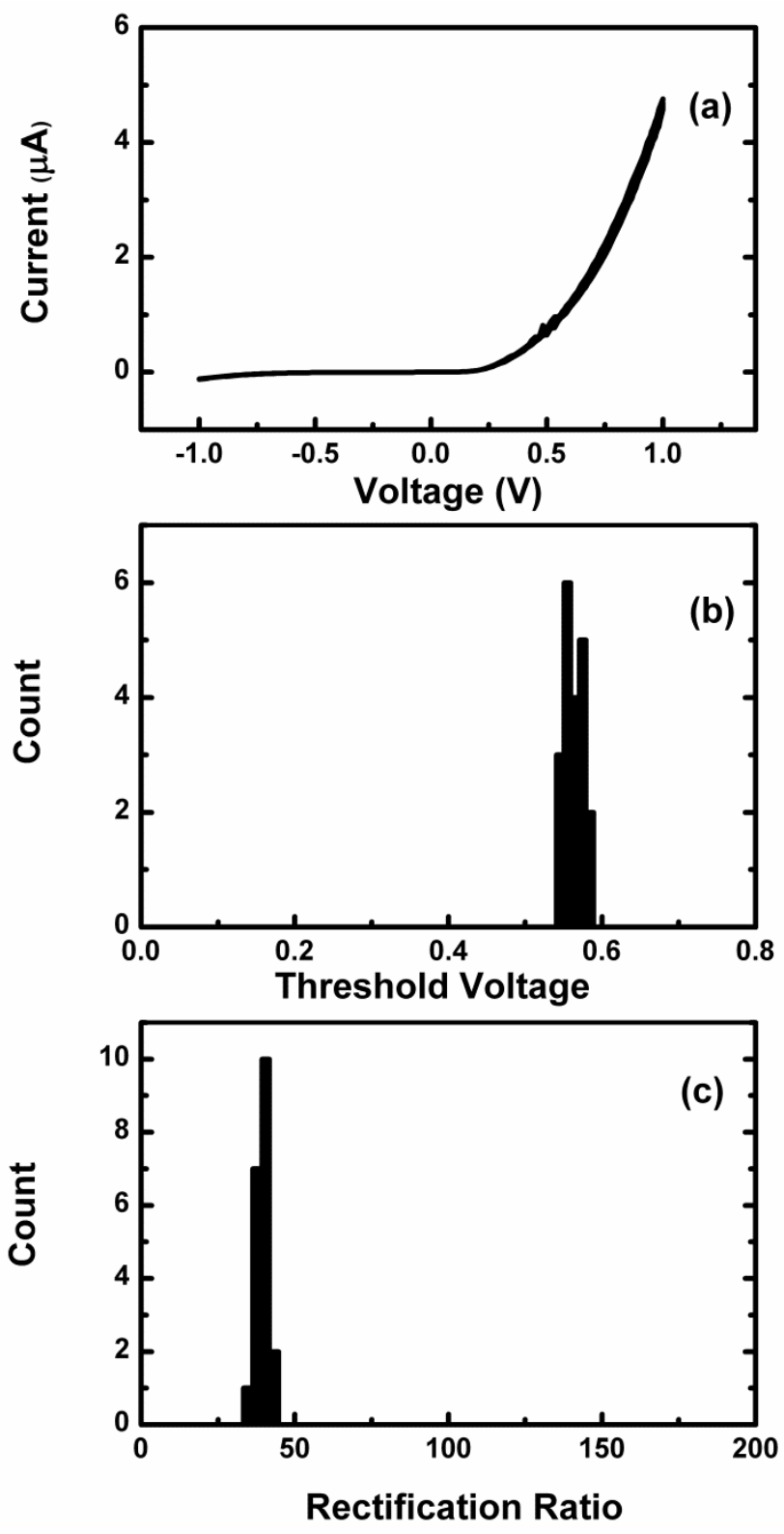

Fig. 1. (a) Ten cyclic IV measurements, (b) a histogram of the threshold voltage, and (c) a histogram of the rectification ratio from drop-dry samples. 
To further examine the stability and reproducibility of the electrical properties of the FeP molecules, the histograms of the threshold voltage and the rectification ratio were constructed for the FeP drop-dry samples. The histogram of the threshold voltage from the drop-dry sample was built from 20 IV measurements from ten cyclic IV curves and is shown in Fig. 1(b). The threshold voltage is centered at $0.56 \mathrm{~V}$ and the values are distributed within a narrow voltage range of $\pm 0.01 \mathrm{~V}$. The histogram of the rectification ratio (RR) of the dropdry sample is also built from the same $20 \mathrm{IV}$ measurements and is shown in Fig. 1(c). The histogram is centered at an RR value of 39 and the values are distributed in a narrow range of \pm 2.46 .
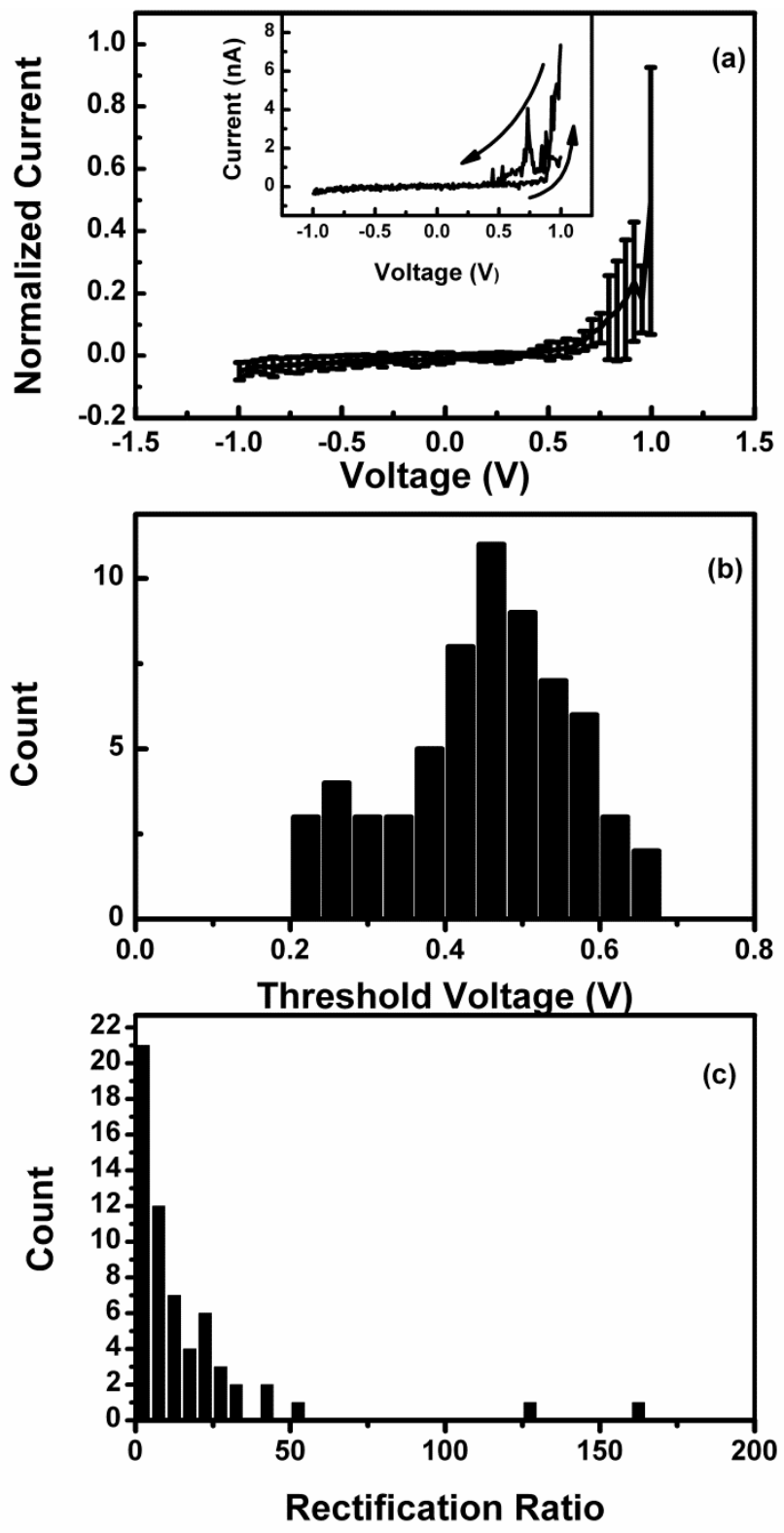
Fig. 2. (a) An average IV curve from eight measurements, (b) a histogram of the threshold voltage, and (c) a histogram of the rectification ratio from samples that were deposited in the FeP toluene solution for 60 seconds. Inset: Fig. 2(a) A characteristic IV measurement. The arrows indicate the direction of the voltage scan.

Similar to the drop-dry sample, rectification from the $60 \mathrm{~s}$ sample was also investigated. A characteristic IV measurement showing rectification is shown in the inset of Fig. 2(a). The voltage was swept from $-1 \mathrm{~V} \rightarrow 1 \mathrm{~V} \rightarrow-1 \mathrm{~V}$. The direction of the scan is denoted with arrows. The current starts to increase drastically after a threshold voltage is reached. In the negative bias region the current is close to zero, which is consistent with the previous measurement for the drop-dry sample. However, the current is in the nano-ampere range compared with the micro-ampere range for the drop-dry sample. When compared to measurements of other molecular junctions [11], the measured current is within the same range, but the rectification ratio is not comparable. Similar to the molecular junctions in our previous work [12], the current is in a different range; however, the rectification ratio is consistent with our results. This may suggest that the rectification ratio depends on the molecule forming the molecular junction, but that the current can change with the size of AFM tip and the number of molecules that is measured in the junction.

For the $60 \mathrm{~s}$ sample, the individual rectification curves fluctuated and this instability worsened with increasing bias. This could arise from the increase in the local temperature induced by local joule heating near the molecule-electrode junction. Assuming bulk heat conduction, the local temperature can be theoretically described as [13]

$$
T_{\text {eff }}=\left(T_{0}^{4}+\gamma^{4} V_{\text {bias }}^{2}\right)^{1 / 4}
$$

where $T_{0}$ is ambient temperature, $\checkmark$ describes the magnitude of the local joule heating effect on $\mathrm{T}_{\text {eff }}$, and $\mathrm{V}_{\text {bias }}$ is the bias applied across the molecular junction. From the above equation the local temperature $\mathrm{T}_{\text {eff }}$ increases with the bias $\mathrm{V}_{\text {bias }}$. A similar observation was reported by another group. [6] An increase in the local temperature contributes to an unstable moleculeelectrode junction, which could explain our experimental observation. The individual rectification curves are also different from each other, which may reflect hybridization changes at the molecule-metal interface [14] or the different contact geometries [15] formed at the molecule-tip and the molecule-substrate interface.

In order to extract additional information from the measurements for the $60 \mathrm{~s}$ sample, a statistical analysis was completed to analyze the data to compare to the drop-dry samples. An average of the normalized IV curve from eight measurements is shown in Fig. 2(a). Because the magnitude of the current was different for each individual IV curve, the curves were normalized. The normalization was completed by dividing the current by the maximum value and then the maximum of the curve was normalized to one. The threshold voltage from the averaged IV curve is $\sim 0.45 \mathrm{~V}$. 
To better describe the rectification from the $60 \mathrm{~s}$ sample, histograms of the threshold voltage and rectification ratio were constructed. The histogram of the threshold voltage was constructed from 65 IV measurements from five samples and is shown in Fig. 2(b). The distribution range of the threshold voltage is from $0.225 \mathrm{~V}$ to $0.675 \mathrm{~V}$ and the most probable value of the threshold voltage occurred near $0.45 \mathrm{~V}$. The distribution of the rectification ratio $(\mathrm{RR})$ is reflected in the histogram constructed from $65 \mathrm{IV}$ curves and is shown in Fig. 2(c). The RR falls in a range from 2.5 to 162.

As a control experiment an IV curve of a bare template-stripped gold substrate was measured before and after submerging the sample in toluene for $60 \mathrm{~s}$. The IVs curves measured were linear and the change in resistance before and after submerging was $51.8 \Omega$. This change in resistance is several orders of magnitude less than that of FeP molecules. Therefore, the effect of toluene on the IV measurement from the FeP can be neglected. The linear behavior of the IV after submerging the sample in the toluene indicates that the rectification observed in the IV is attributed to the FeP molecules deposited on the substrate. 

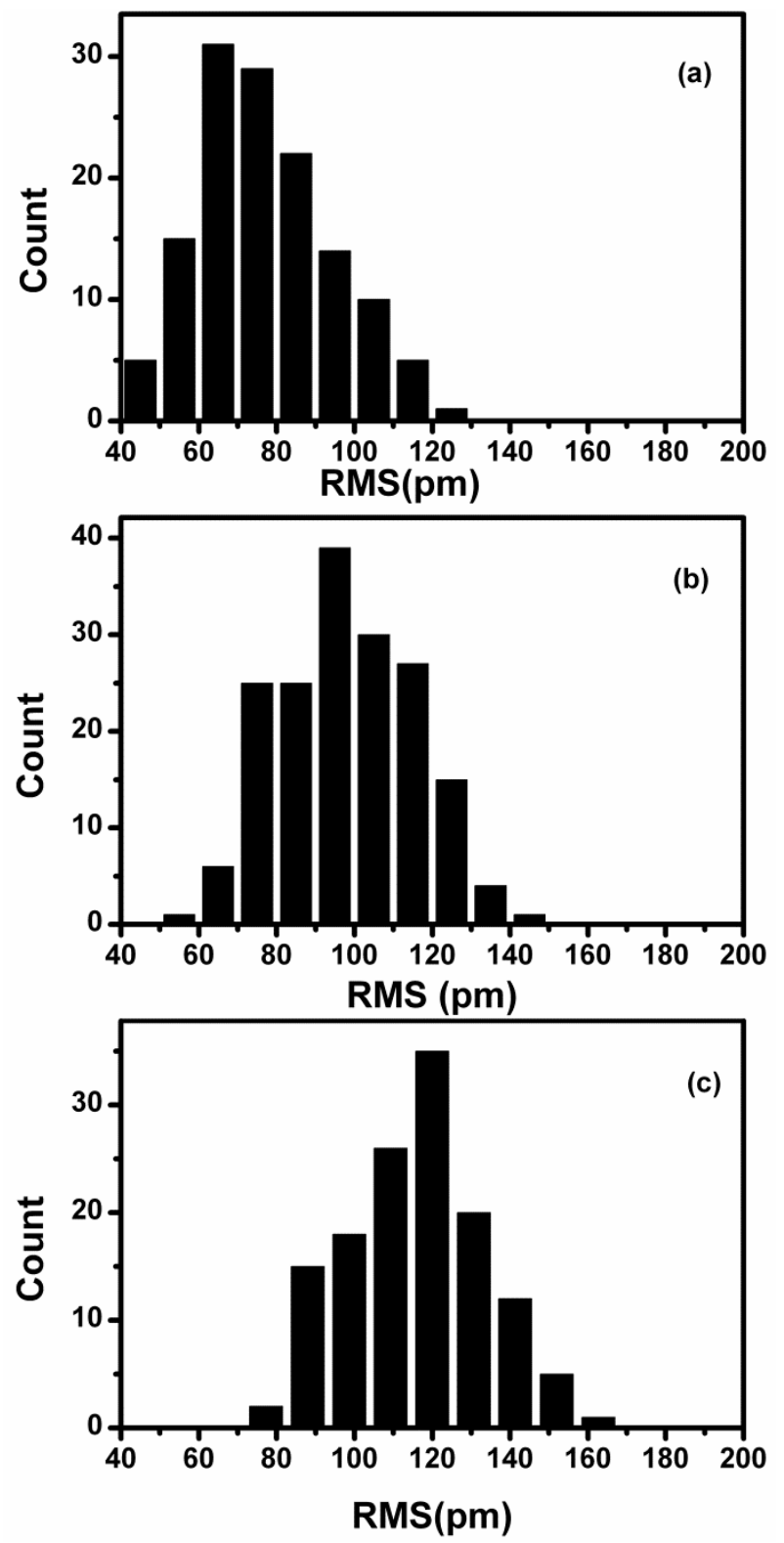

Fig. 3. Histograms of the roughness (RMS) from (a) the template-stripped gold substrate, (b) the drop-dry sample, and (c) the 60 second sample. The scan size was $0.05 \mu \mathrm{m} \times 0.05 \mu \mathrm{m}$.

The explanation for the rectification has been discussed in previous work [12]. This explanation is based on a model of the alignment of energy levels between the molecule and electrode (i.e. tip or substrate). In the model, the threshold voltage is determined by the energy difference between the Fermi level of the electrodes and the molecular orbital level. The experiment was performed at room temperature which leads to thermal broadening of the molecular energy level. The distribution of the threshold voltage measured from the IV curves for the $60 \mathrm{~s}$ sample may be related to the thermal broadening of the molecular energy level. The shift of the threshold voltage for different IV measurements may be an intrinsic property of the molecule at room temperature. 
Based on the IV measurements, the rectification observed in the IV measurement from the drop-dry sample is stable and reproducible. This is also reflected in the analysis of the histogram of the threshold voltage and rectification ratio. The distribution of the histograms for both the threshold voltage and rectification ratio for the drop-dry sample is more confined than the distributions for the $60 \mathrm{~s}$ sample. In order to investigate the mechanism for both stability and reproducibility observed in the IV curves, the topography of samples were examined.

The topography of a bare template-stripped gold substrate, the $60 \mathrm{~s}$ sample of FeP, and the drop-dry sample of FeP was examined by AFM in contact mode (see Supporting Information Fig. S1). The histograms of the root mean square (RMS) values from the samples were constructed from 132, 173, and 134 sample areas of size $0.05 \mu \mathrm{m} \times 0.05 \mu \mathrm{m}$, from the template-stripped gold substrate, the drop-dry sample, and the $60 \mathrm{~s}$ sample, respectively. Smaller areas of $0.05 \mu \mathrm{m} \times 0.05 \mu \mathrm{m}$ were selected from larger scan areas of either $1 \mu \mathrm{m} \times 1$ $\mu \mathrm{m}$ or $2 \mu \mathrm{m} \times 2 \mu \mathrm{m}$. The scans were taken from at least four samples for each of the three histograms. The histograms are shown in Fig. 3. The average of the RMS value from the template-stripped gold, drop-dry sample, and $60 \mathrm{~s}$ sample is $76.8 \mathrm{pm} \pm 17.2 \mathrm{pm}, 97.9 \mathrm{pm} \pm$ $17.3 \mathrm{pm}$, and $115.7 \mathrm{pm} \pm 17.5 \mathrm{pm}$, respectively, which indicates that the $60 \mathrm{~s}$ sample is rougher than the drop-dry sample. One explanation for the difference in roughness between the drop-dry sample and the $60 \mathrm{~s}$ sample is that it may be caused by different arrangements of the contact geometries at the molecule-electrode interface (i.e. tip or gold substrate). In a previous study [17] a deposition technique involving direct adsorption assumed the molecules to be self-assembled or nearly vertically aligned to the gold substrate. FeP molecules of our 60s samples were directly deposited on the gold substrate in similar way, and assumed to be nearly vertically aligned to the substrate. The fluctuation in the IV curves from the $60 \mathrm{~s}$ sample observed is similar to experiments in which the molecules are vertically aligned on the surface [6]. However, for the drop-dry sample a different deposition method from the $60 \mathrm{~s}$ sample has been used, and stable and reproducible electrical properties have been observed, which may suggest the molecules from the drop-dry samples have a different orientation from the molecules for the $60 \mathrm{~s}$ samples. If the molecules from the drop-dry samples have an arrangement on the gold substrate with the porphyrin ring parallel to the surface, then the thermal contact area of the molecule with the substrate increases. This thermal contact at the molecule-electrode interface could reduce the phonon mismatch so as to enhance the thermal conductance, minimize the local heating, and reach a sufficient electrical durability as observed in our experiment and suggested by the other reports [16].

\section{Conclusion}

In conclusion, in this experiment we have observed rectification behavior from both a dropdry sample and a $60 \mathrm{~s}$ deposition sample of FeP. The rectification observed in the IV from the drop-dry sample was stable and reproducible. The distribution in the histogram for both the threshold voltage and rectification ratio for the drop-dry sample is more confined than that for 
the $60 \mathrm{~s}$ sample. The stability and reproducibility of the rectification observed has been attributed to the good thermal contact between the molecules and the AFM tip based on roughness (RMS) analysis of AFM topography of the samples.

\section{Acknowledgments}

This work was partially supported by the National Science Foundation DMR\# 1150866 and the New York State's Empire State Development's Division of Science, Technology, Innovation (NYSTAR) Contract \#C100117 and Contract \#C130117. The authors acknowledge Han Wang for discussions.

\section{Reference}

1. Arieh Avira, Mark A. Ratner, Chem. Phys. Letts. 29(1974)277.

2. N.J.Geddes, J.R.Sambles, D.J.Jarvis, W.G.Parker, and D.J.Sandman, Appl. Phys. Letts. 56 (1990)1916.

3. Robert M. Metzger, Bo Chen, Ulf Hopfner, M. V. Lakshmikantham, Dominique Vuillaume, Tsuyoshi Kawai, Xiangli Wu, Hiroaki Tachibana, Terry V. Hughes, Hiromi Sakurai, Jeffrey W. Baldwin, Christina Hosch, Michael P. Cava, Ludwig Brehmer, and Geoffrey J. Ashwell, J. Am. Chem. Soc. 119(1997)10455.

4. A. Dhirani, P.-H. Lin, and P. Guyot-Sionneest, J. Chem. Phys. 106(1997)5249.

5. Kent S. Alleman, Kara Weber, and Stephen E. Creager, J. Phys. Chem. 100 (1996)17050.

6. Ismael Diez-Perez, Joshua Hihath, Youngu Lee, Luping Yu, Lyudmyla Adamska, Mortko A. Kozhushner, Ivan I. Oleynik and Nongjian Tao, Nature Chem. 1(2009)635.

7. Andreas Stabel, Peter Herwig, Klaus Mullen, and Jurgen P. Rabe, Angew. Chem. Int. Ed. Engl. 34(1995)1609.

8. Kun Wang, Jiangfeng Zhou, Joseph M. Hamill, and Bingqian Xu, J. Chem. Phys. 141 (2014)054712.

9. E. D. Mentovich, I.Kalifa, A. Tsukernik, A. Caster, N. Rosenberg-Shraga, H. Marom, M. Gozin, S. Richter, Small 4(2008)55.

10. Martin Hegner, Peter Wagner, Giorgio Semenza, Surface Science 291(1993)39.

11. G.J. Ashwell, B. Urasinska, W.D,Tyrrell, Phys, Chem. Chem, Phys, 8 (2006)3314.

12. Xiaoyu Wang and Kim. M. Lewis, Mater. Chem. Phys. 136(2012)190.

13. Zhifeng Huang, Bingqian Xu, Yuchang Chen, Massimiliano Di Ventra, and Nongjian Tao, Nano Letts. 6(2006)1240.

14. A. M. Moore; A. A. Dameron; B. A. Mantooth; R. K. Smith; D. J. Fuchs; J. W. Ciszek; F. Maya; Y. X. Yao; J. M. Tour; P. S. Weiss, J. Am. Chem. Soc. 128( 2006)1959. 
15. S. Y. Quek, M. Kamenetska, M. L. Steigerwald, H. J. Choi, S. G. Louie, M. S. Hybertsen, J. B. Neaton, L. Venkataraman, Nat. Nanotechnol. 4 (2009)230.

16. Akusu Tsutsui, Masateru Taniguchi, and Tomoji Kawai, Nano Letts. 8(2008)3293.

17. A. Singh, D. H. Dahanayaka, A. Biswas, L. A. Bumm, and R. L. Halterman, Langmuir 26(2010)13221. 